\title{
Oligodendrogliomas: a genética molecular e o desenvolvimento de estratégia terapêutica adjuvante
}

Clovis Orlando da Fonseca', Juliana de Saldanha da Gama Fischer ${ }^{2}$, Marcos Masini, Débora Futuro ${ }^{4}$, Regina Caetano ${ }^{5}$, Cerli R. Gattass ${ }^{6}$, Thereza Q. Santos ${ }^{7}$

\author{
Faculdade de Medicina da Universidade Federal Fluminense, Rio de Janeiro, RJ, Brasil \\ Instituto de Química da Universidade Federal do Rio de Janeiro, RJ, Brasil \\ Faculdade de Medicina do Planalto Central, Brasília, DF, Brasil \\ Faculdade de Farmácia da Universidade Federal Fluminense, Rio de Janeiro, RJ, Brasil \\ Instituto de Biofísica da Universidade Federal do Rio de Janeiro, RJ, Brasil \\ Instituto de Biologia da Universidade Federal Fluminense, Rio de Janeiro, RJ, Brasil
}

\section{RESUMO}

A incidência dos oligodendrogliomas tem aumentado provavelmente em razão do progresso na precisão de diagnóstico. Aproximadamente dois terços dos pacientes com a forma mais agressiva, oligodendroglioma anaplásico, mostram resposta substancial à quimioterapia com a associação procarbazina/lomustina/ vincristina (PCV). Entretanto, os resultados da quimioterapia com PCV para oligodendrogliomas anaplásicos recidivados têm sido limitados. A progressão anaplásica dos oligodendrogliomas resulta em grande número de células com ERK/MAPK ativadas. O monoterpeno álcool perílico demonstra atividades quimiopreventiva e quimioterápica em diversos modelos de tumores e sugere-se que estas possam estar associadas com a capacidade de inibir a farnesilação pós-traducional e a sinalização da Ras, assim como a cascata de sinalização por meio da RAF-MEK-ERK. Estudo do nosso grupo observou que pode estar atuando mediante a inibição da fosforilação da extracellular regulated kinase (ERK), uma proteína envolvida na cascata de transdução de sinal através da membrana e proliferação celular induzida pela proteína Ras. Este artigo discute a redução de oligodendroglioma anaplásico recidivado em paciente tratado durante nove meses com álcool perílico por via intranasal.

\section{PALAVRAS-CHAVE}

Oligodendroglioma. Álcool perílico. Quimioterapia. Monoterpenos.

\section{ABSTRACT}

Oligodendrogliomas: molecular genetics and development of adjuvant therapeutic strategies The incidence of oligodendrogliomas is increasing, most likely due to more accurate diagnosis. Approximately two thirds of patients with the more aggressive form - anaplastic oligodendrogliomas have shown substantial response to first-line procarbazine/lomustine/vincristine (PCV) therapy. However, experience with chemotherapy for relapsing anaplastic oligodendrogliomas following PCV therapy is limited. Anaplastic progression in oligodendrogliomas results in a larger number of cells with active ERK/MAPK. Perillyl alcohol $(\mathrm{POH})$ displays preventive and therapeutic activity against a wide variety of tumor models and it has been suggested that this might be associated with the ability to inhibit post translational farnesylation and signaling from Ras, as well as, signaling through RAF-MEK-ERK cascade. Study of our group observed that $\mathrm{POH}$ seems to act inhibiting extracellular regulated kinase (ERK), a protein evolved in signal transduction signaling through plasmatic membrane and cellular proliferation induced by Ras protein. This article discusses the reduction of recurrent anaplastic oligodendroglioma in a patient treated with intranasal administration of $\mathrm{POH}$ after nine months.

\section{KEY WORDS}

Oligodendroglioma. Perillyl alcohol. Chemotherapy. Monoterpenes.

\footnotetext{
1 Professor adjunto do Serviço de Neurocirurgia da Faculdade de Medicina da Universidade Federal Fluminense.

2 Doutoranda do Laboratório de Química de Proteína do Instituto de Química da Universidade Federal do Rio de Janeiro.

3 Professor adjunto do Serviço de Neurocirurgia da Faculdade de Medicina do Planalto Central.

4 Professora adjunta da Faculdade de Farmácia da Universidade Federal Fluminense.

5 Professora adjunta do Departamento de Biologia Celular e Molecular do Instituto de Biologia da Universidade Federal Fluminense. 6 Professora adjunta do Laboratório de Imunologia Celular do Instituto de Biofísica da Universidade Federal do Rio de Janeiro.

7 Professora titular do Departamento de Biologia Celular e Molecular do Instituto de Biologia da Universidade Federal Fluminense.
} 


\section{Introdução}

A classificação da Organização Mundial da Saúde define oligodendrogliomas como tumores difusamente infiltrativos e os divide em: oligodendrogliomas de grau II e oligodendrogliomas anaplásicos de grau III $^{27}$. Um estudo demonstra que a incidência ${ }^{28}$ desses tumores tem alcançado $25 \%$ entre os gliomas ${ }^{10}$. Os oligodendrogliomas de grau II acometem pacientes com idade em torno de 40 anos, enquanto os anaplásicos ocorrem em pacientes acima de $55 \operatorname{anos}^{34}$. A sua etiologia não está esclarecida, sendo descritos casos de contusão cerebral prévia ${ }^{41}$, secundários à irradiação de adenomas pituitários ${ }^{24}$, e ocorrência familiar de oligodendrogliomas polimorfos ${ }^{29}$. Os tumores oligodendrogliais vêm despertando interesse entre os neuroncologistas nos últimos anos, em razão da elevada resposta à quimioterapia e da possibilidade de dividi-los em subgrupos com base no estudo de sua biologia molecular ${ }^{12}$. A caracterização das alterações genéticas desses tumores ${ }^{49}$ (figura 1) promete ser tão importante quanto os achados histológicos e os fatores clínicos na definição da terapia apropriada $^{54}$. Especificamente, diversos estudos têm confirmado que a deleção dos cromossomos $1 \mathrm{p}$ e 19q está associada com o fenótipo desses tumores e com a longa sobrevida sem recorrência após radioterapia e/ou quimioterapia ${ }^{51}$.

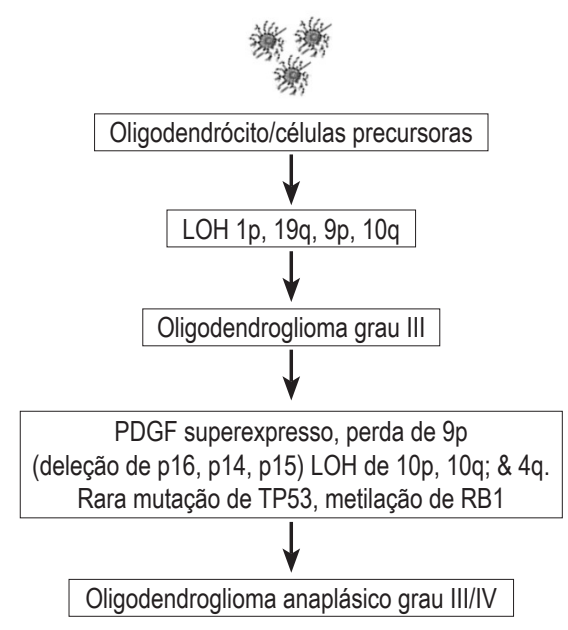

Figura 1 - Alterações genéticas envolvidas no desenvolvimento dos oligodendrogliomas.

\section{Tratamento dos tumores oligodendrogliais}

O tratamento convencional dos oligodendrogliomas consiste em cirurgia, radioterapia e quimioterapia.
A abordagem cirúrgica visa à ressecção total, o que permite a descompressão do efeito de massa, com conseqüente aumento da sobrevida, e a avaliação anatomopatológica que permite a classificação do grau do tumor. A radioterapia convencional é realizada com doses de 45Gy a 65Gy, após o tratamento cirúrgico ${ }^{21}$. Autores relatam claro benefício com esse tratamento ${ }^{32}$, enquanto outros demonstram que não há efeito significativo no aumento da sobrevida ${ }^{11}$. Um estudo propõe a radiocirurgia na ocorrência de oligodendrogliomas recidivados como uma segunda linha de tratamento, porém ressalta a necessidade de pesquisas prospectivas para elucidar as indicações dessa técnica ${ }^{31}$. Deve-se levar sempre em consideração que pacientes com tumores oligodendrogliais apresentam expectativa de sobrevida de alguns anos e que a radioterapia pode induzir lesões como leucoencefalite. Pesquisas recomendam adiar a radioterapia em oligodendrogliomas de baixo grau até a progressão para a forma anaplásica, porque concluem que oligodendroglioma maligno é o único tumor glial sensível à quimioterapia ${ }^{8}$. Em 1988, Cairncross e MacDonald ${ }^{7}$ relataram a resposta dos oligodendrogliomas anaplásicos à quimioterapia. Posteriormente a esse estudo, o uso da combinação procarbazina/lomustina/ vincristina denominada PCV mostrou ser a mais efetiva combinação de quimioterápicos. Aproximadamente $75 \%$ dos pacientes com oligodendroglioma anaplásico respondem a esse tratamento. Os oligoastrocitomas também parecem responder bem a essa conjunção de drogas $^{30}$. Contudo, estudos demonstram que pacientes portadores de oligodendroglioma anaplásico não apresentam aumento da sobrevida após tratamento quimioterápico com PCV, independentemente do grau da ressecção cirúrgica efetuada ${ }^{17}$. Temozolamida tem sido introduzida como opção de tratamento para pacientes com oligodendroglioma de grau $\mathrm{II}^{22}$ e como última opção para os pacientes com a forma anaplásica ${ }^{13}$. Entretanto, o sucesso do tratamento quimioterápico, com prolongamento da sobrevida, está associado ao aumento da incidência de leucemia secundária ${ }^{42}$.

\section{Patogênese molecular dos tumores oligodendrogliais}

Os oligodendrogliomas apresentam perda combinada dos alelos no braço curto do cromossomo 1 (1p) e no braço longo do cromossomo 19 (19q). Essas alterações cromossomais são encontradas em $90 \%$ dos oligodendrogliomas ${ }^{3}$. A alta freqüência dessas deleções aumenta a possibilidade futura de classificação desses tumores pela sua alteração molecular, melhor que pela sua morfologia. Nos oligodendrogliomas anaplásicos 
é menor a freqüência de LOH (loss of heterozygosity) $1 \mathrm{p} / 19 \mathrm{q}$ (a perda de heterozigose é a perda de um alelo de um gene supressor). Existe proporcionalidade entre a LOH de 1 p e 19q desses tumores e a sua localização anatômica nos lobos frontal, parietal e occipital. Os tumores que não apresentam deleção de ambos os alelos predominantemente se localizam no lobo tempora $l^{54}$. Outro estudo analisando comparativamente oligodendrogliomas e oligoastrocitomas identificou semelhante localização anatômica ${ }^{38}$. Entretanto, para os tumores astrocíticos nenhuma proporcionalidade foi observada entre o espectro mutacional e a sua localização anatômica ${ }^{38}$.

Os genes supressores de tumor mapeiam as regiões cromossomais com freqüentes perdas dos alelos ${ }^{25}$. Portanto, múltiplas deleções estudadas em localizações de cromossomos e inúmeras regiões foram identificadas. $\mathrm{O}$ mapeamento das deleções nas regiões do cromossomo $1 p$ foi feito nos raros oligodendrogliomas com deleções intersticiais. Três regiões diferentes foram identificadas no $1 \mathrm{p}$, indicando o envolvimento de múltiplos genes supressores tumorais nos tumores oligodendrogliais: 1p36.3, $1 \mathrm{p} 36.1-2$ e $1 \mathrm{p} 34.35^{3}$.

Os pacientes portadores de oligodendrogliomas respondem melhor à quimioterapia e à radioterapia do que os pacientes com astrocitomas. Embora o mecanismo preciso que regula a sensibilidade à quimioterapia e à radioterapia dos oligodendrogliomas não esteja ainda esclarecido, as evidências de diversos estudos retrospectivos sugerem que a perda de alelos de $1 \mathrm{p} / 19 \mathrm{q}$ é um marcador da resposta para a terapia e a sobrevida prolongada dos pacientes com esses tumores ${ }^{2,3}$. Apesar de ainda sujeitas à confirmação por estudos prospectivos, essas observações são muito intrigantes e certamente sublinham a importância da estratificação de pacientes com oligodendrogliomas, com e sem deleção de 1p/19q. É possível que as regiões $1 p$ ou 19q, ou ambas, contenham os genes associados com o desenvolvimento de tumores oligodendrogliais e esteja aí a resposta positiva para a quimioterapia e radioterapia de pacientes com oligodendrogliomas.

\section{Oncogenes}

Existem poucos relatos na literatura sobre a expressão dos protoncogenes nos oligodendrogliomas humanos, sendo raras as suas mutações. Entretanto, a expressão dos receptores tirosina-quinases que ativam as vias $\mathrm{PI} 3 \mathrm{~K} / \mathrm{AKT}$ e RAS/MAPK é freqüentemente encontrada. A análise dessas vias promotoras de células transformadas é extremamente importante. O receptor do fator de crescimento (EGFR) está freqüentemente amplificado e superexpresso nos astrocitomas de alto grau $^{27}$. Nos oligodendrogliomas, o aumento da expressão do mRNA e da proteína do EGFR foi observado em $80 \%$ dos $\operatorname{casos}^{5,36}$. Os mesmos níveis de expressão foram encontrados nos oligodendrogliomas de graus II e III, indicando que essa alteração é um evento inicial na tumorigênese dos oligodendrogliomas ${ }^{23}$. O aumento da expressão do fator de crescimento derivado de plaquetas (PDGFR-A) e seu receptor PDGFR- $\alpha$ foram verificados em $94 \%$ dos oligodendrogliomas de graus II e III, sugerindo estimulações autócrina e parácrina ${ }^{44}$. A superexpressão desses fatores de crescimento estimula a via de transdução do sinal através da membrana induzida pela proteína Ras. Uma vez que a atividade de Ras é dependente da farnesilação, uma modificação pós-traducional dessa proteína catalisada pela enzima farnesiltransferase (figura 2) pode ser vista como alvo promissor para a estratégia terapêutica dos gliomas malignos. Portanto, os agentes inibidores da farnesiltransferase que bloqueiam a atividade da Ras e a conseqüente cascata de sinalização podem ser considerados promissores agentes quimioterápicos para esses tumores ${ }^{45}$.

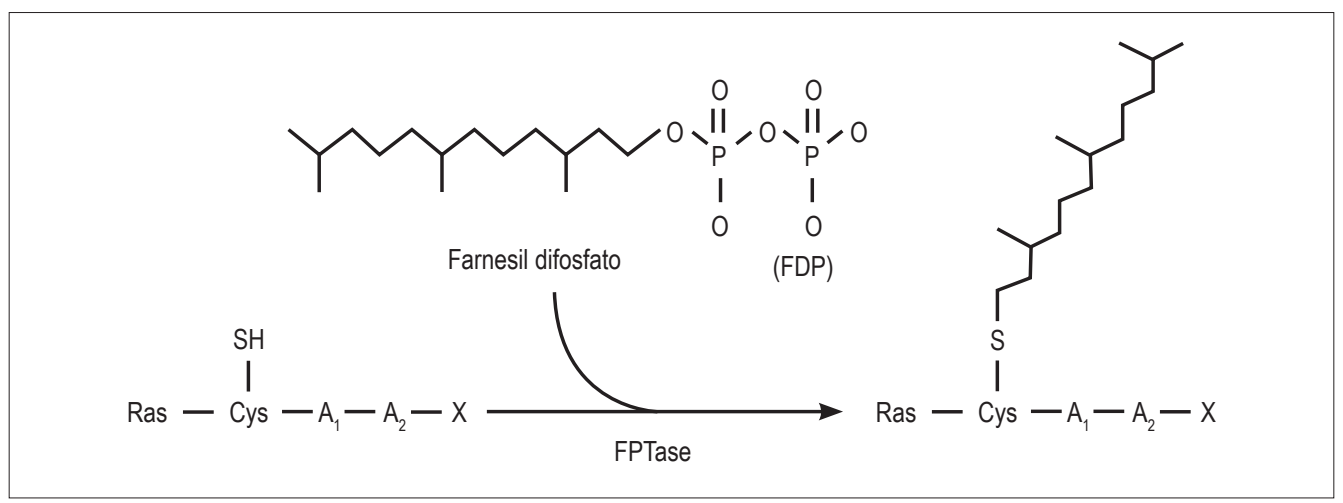

Figura 2 - Farnesilação da proteína Ras. A enzima farnesiltransferase (FPTase) catalisa a adição do grupo farnesil à cisteína localizada a três aminoácidos da região carboxiterminal da cadeia polipeptídica. (Ilustração modificada de Rowinsky EK, Windle JJe Von Hoff DD ${ }^{45}$.) 


\section{Álcool perílico e sua atuação em âmbito molecular}

O álcool perílico (figura 3), também chamado p-meta-1,7-dieno-6-ol ou 4-isopropenilciclo-hexenocarbinol, é um monoterpeno natural monocíclico hidroxilado, isolado dos óleos essenciais de lavanda, saliva, hortelã, menta, cerejas, sementes de aipo e várias outras plantas ${ }^{1}$. Estudos em animais têm mostrado que o álcool perílico é um agente quimioterápico eficaz na regressão de tumores de mama ${ }^{20}$, pâncreas ${ }^{6,47}$, fígado ${ }^{37}$, próstata $^{9}$ e agente quimiopreventivo nos tumores de cólon $^{43}$, melanomas ${ }^{35}$ e neuroblastomas ${ }^{46}$.

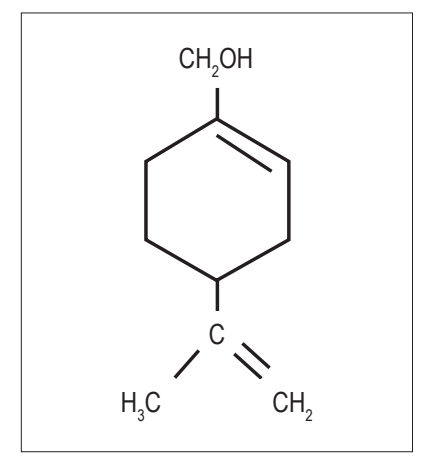

Figura 3 - Estrutura química do álcool perílico.

Descreve-se que o álcool perílico atua na inibição da transdução de sinal através de membrana por inibir a farnesilação da proteína Ras ${ }^{1,6,37}$. As proteínas Ras desempenham função importante na transdução de sinais de fatores de crescimento. Elas se tornam funcionais após sofrerem uma modificação pós-traducional, chamada farnesilação, a qual permite a sua ancoragem à superfície interna da membrana plasmática ${ }^{45}$. A ativação das proteínas Ras leva a uma cascata de fosforilações envolvendo serina e treonina cinases (RAF) e cinases ativadas por mitógenos (MAPK), que são responsáveis pela fosforilação de uma variedade de proteínas citosólicas e nucleares, incluindo outras proteínas cinases e fatores de transcrição ${ }^{4}$. Em recentes experimentos realizados pelo nosso grupo em células de adenocarcinoma de pulmão humano (A549) tratadas com álcool perílico e a utilização de precursor radioativo $\left(\right.$ Met- $\left.\mathrm{S}^{35}\right)$ com posterior fracionamento das proteínas por meio de eletroforese unidimensional, foi possível observar proteínas diferencialmente expressadas nas células tratadas quando comparadas com células controle. Pela primeira vez se verificou uma nova via de ação do álcool perílico na transdução de sinal através de membrana. Uma das etapas de controle em que o álcool perílico pode estar atuando é por meio da inibição da fosforilação da extracellular regulated kinase (ERK), uma proteína envolvida na cascata de transdução de sinal através de membrana ${ }^{14}$.

\section{Caso ilustrativo}

Paciente do sexo feminino, 68 anos, apresentou quadro de crises convulsivas e cefaléia frontal em junho de 1999. O exame neurológico foi normal, com índice de Karnofsky de 90. A ressonância magnética (RM) revelou presença de processo expansivo na região parietal direita. Realizou-se craniotomia frontoparietal direita para ressecção total do tumor, o que foi confirmado pelo exame de RM pós-operatório. $\mathrm{O}$ exame anatomopatológico evidenciou astrocitoma de grau II. Houve recorrência da lesão, após cerca de três anos, em julho de 2002. Submeteu-se à nova craniotomia para exérese do tumor recidivado. O diagnóstico de oligodendroglioma anaplásico foi, desta vez, confirmado pelo Serviço de Anatomia Patológica da Emory University, em Atlanta, EUA. Após o tratamento cirúrgico, foi submetida à radioterapia (59,4 Gy). Permaneceu assintomática até outubro de 2004, quando procurou novamente o serviço de neurocirurgia com queixas de cefaléia e tonteira. O exame neurológico evidenciou hemiparesia esquerda e a paciente apresentava incontinência urinária. A RM revelou presença de tumor recidivado com localização frontoparietal direita, com compressão do ventrículo homolateral. Iniciou-se quimioterapia com temozolomida (150 a $200 \mathrm{mg} / \mathrm{m}^{2} /$ dia, durante 5 dias, em ciclo de 28 dias). A paciente apresentou náuseas, vômitos, tonteira e os exames laboratoriais revelaram contagem de plaquetas de $99.000 / \mathrm{mm}^{3}$. Após a retirada de temozolamida, a paciente foi incluída no protocolo do Estudo Clínico Fase II do Álcool Perílico em pacientes com gliomas malignos recidivados (CONEP 9.681 no 25000.009267/200425 , aprovado em julho de 2004). O álcool perílico vem sendo administrado em concentração de $0,3 \%$, por via inalatória, quatro vezes ao dia, desde 20 de maio de 2005. Os exames de sangue e urina e radiografia de tórax não mostram sinais de toxicidade. A paciente apresenta-se lúcida, sem déficit motor e distúrbios esfincterianos. Vem fazendo uso de medicação anticonvulsivante e foi suspensa a corticoterapia. A avaliação do volume tumoral vem sendo feita com exames de RM que mostraram, em fevereiro de 2006, redução significativa do tumor (figura 4). 


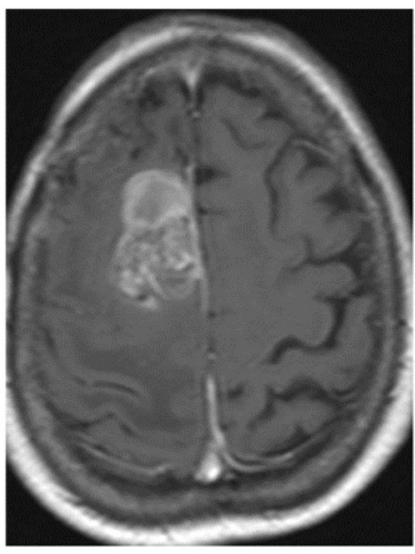

A

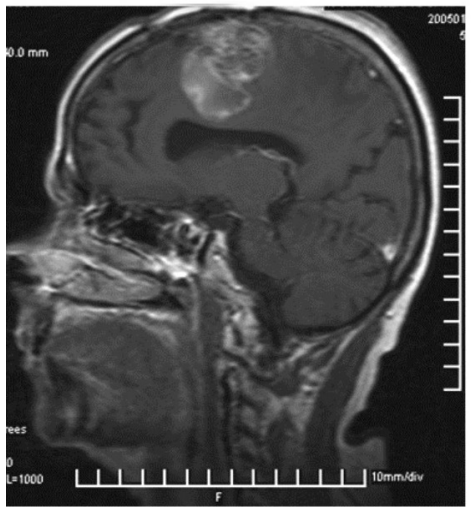

D

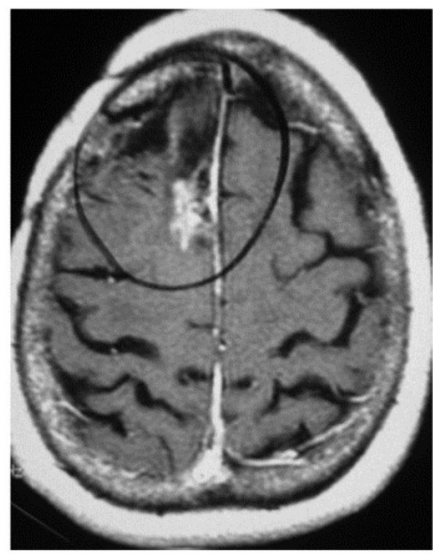

B

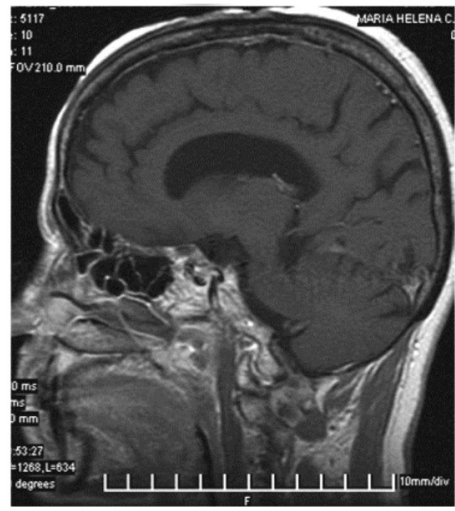

E

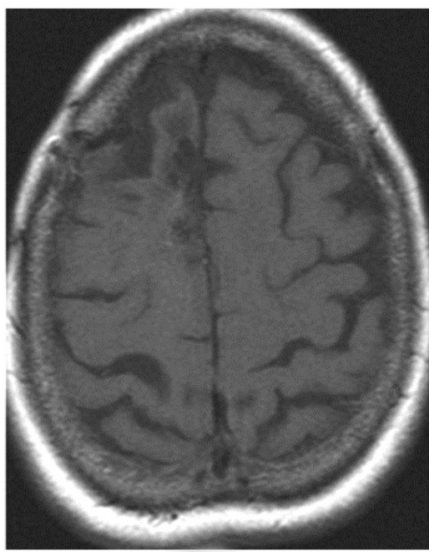

C

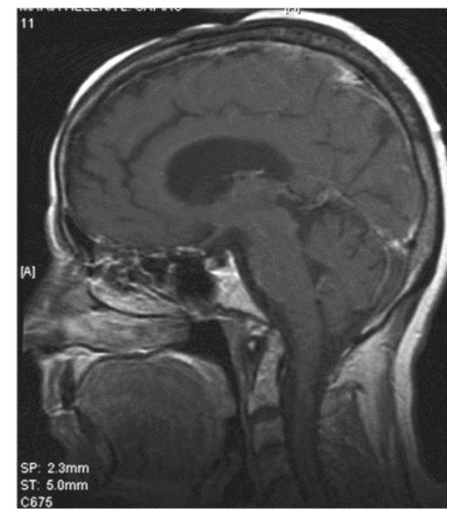

Figura 4 - A e D) RM antes do início do tratamento com álcool perílico; $B$ e E) após cinco meses de tratamento; Ce F) após nove meses de tratamento com álcool perílico administrado por via intranasal.

\section{Discussão}

Os oligodendrogliomas, assim como outros tumores gliais, têm como característica a infiltração pelo tecido cerebral. A extensão da infiltração não é detectada de forma precisa pelos exames de imagem correntes. Portanto, apesar da importância do tratamento cirúrgico, os pacientes com oligodendrogliomas são raramente curados pela ressecção cirúrgica, mesmo se todo o tumor identificável for removido ${ }^{19}$. As recidivas são usuais no sítio operatório, ocorrendo após alguns anos em pacientes portadores de tumores de baixo grau, às vezes transformados na forma anaplásica ${ }^{33}$. Estudos não demonstram benefícios da radioterapia adjuvante nesses pacientes e enfatizam que a radioterapia produz neurotoxicidade em um terço dos pacientes, o que justifica procrastinar esse tratamento até quando clinicamente necessário ${ }^{39}$. Entretanto, outros autores preconizam radioterapia, especialmente se o tumor recidivado foi ressecado parcialmente. Gannett e cols. ${ }^{19}$ demonstra- ram que os pacientes tratados com cirurgia seguida de radioterapia apresentaram sobrevida de 84 meses, enquanto os que foram tratados somente com cirurgia sobreviveram 47 meses $^{19}$. Concernente ao uso de quimioterapia para oligodendrogliomas de baixo grau, o tratamento com PCV tem sido feito antes ou depois da radioterapia ${ }^{40}$. Muitos neuroncologistas estão administrando PCV antes da radioterapia, isto é, quimioterapia neo-adjuvante, a fim de retardar os efeitos adversos da radiação ${ }^{8}$. Assim como à radiação, à quimioterapia podem associar-se diversos efeitos colaterais. Estudos têm relatado diversos efeitos colaterais com o uso de PCV: náusea, constipação, hepatotoxicidade, anorexia, crises convulsivas, hemorragia intracraniana, neutropenia, trombocitopenias e pneumonia ${ }^{18,50}$. Recorrência dos oligodendrogliomas usualmente ocorre em aproximadamente 12 a 34 meses $^{48}$, e não encontramos relatos de que um segundo ciclo de PCV seja benéfico para esses pacientes. Temozolamida, agente alquilante de segunda geração, está sendo testado em ensaios clínicos como alternativa para a terapia com $\mathrm{PCV}^{48}$. 
Pesquisas indicam o uso da radioterapia pós-operatória para pacientes com oligodendroglioma anaplásico ${ }^{32}$, embora outros estudos não comprovem benefícios neste tratamento ${ }^{53}$. A quimioterapia com PCV tem se mostrado efetiva e é correntemente usada para tratamento desses tumores ${ }^{26}$. Outros autores preconizam que a combinação da radioterapia e quimioterapia com PCV é presumivelmente superior a qualquer terapia isolada ${ }^{48}$. Um estudo recente mostra que, embora os oligodendrogliomas sejam tumores sensíveis à quimioterapia, essa terapêutica adjuvante clássica não melhora a sobrevida nos pacientes com oligodendroglioma anaplásico recorrente ${ }^{51}$. Veninga e cols..$^{52}$ concluíram que a re-irradiação pode ser considerada uma opção de tratamento para essas recidivas em pacientes em boas condições clínicas ${ }^{52}$. Temozolamida tem sido usada para pacientes com oligodendroglioma anaplásico recidivado com relativo sucesso ${ }^{8}$.

Estudos em animais têm mostrado que o álcool perílico é um agente quimioterápico eficaz na regressão de tumores de mama $^{20}$, pâncreas $^{6,47}$, figado ${ }^{37}$, próstata $^{9} \mathrm{e}$ agente quimiopreventivo nos tumores de cólon ${ }^{43}$, melanomas $^{35} \mathrm{e}$ neuroblastomas ${ }^{46}$. Descreve-se que o álcool perílico atua na inibição da transdução de sinal através da membrana por inibir a farnesilação da proteína Ras ${ }^{1,6,37}$. As proteínas Ras desempenham função importante na transdução de sinais de fatores de crescimento, visto que se tornam funcionais após sofrerem uma modificação pós-traducional, chamada farnesilação, a qual permite a sua ancoragem à superfície interna da membrana plasmática ${ }^{45}$. A ativação das proteínas Ras leva a uma cascata de fosforilações envolvendo serina e treonina-cinases como RAF e cinases ativadas por mitógenos (MAPK), que são responsáveis pela fosforilação de uma variedade de proteínas citosólicas e nucleares, incluindo outras proteínas cinases e fatores de transcrição ${ }^{4}$. Nosso grupo demonstrou que o monoterpeno álcool perílico tem efeitos na proliferação celular, na migração, na angiogênese e induz apoptose em linhagens celulares e células de explantes de gliomas malignos ${ }^{15}$. Também foram observadas, por meio de eletroforese unidimensional, proteínas expressas diferencialmente nas células tratadas com álcool perílico quando comparadas com células-controle ${ }^{14}$. Pela primeira vez, verificou-se uma nova via de ação desse álcool na transdução de sinal através da membrana ${ }^{16}$. Uma das etapas de controle em que o álcool perílico pode estar atuando é mediante a inibição da fosforilação da extracellular regulated kinase (ERK), uma proteína envolvida na cascata de transdução de sinal através da membrana e proliferação celular induzida pela proteína Ras ${ }^{14}$. Estudos fase II com o álcool perílico estão em andamento em pacientes portadores de tumores de próstata refratários à terapia hormonal, pulmões, ovário e colorretal. As doses usadas variam de $4.800 \mathrm{mg} / \mathrm{m}^{2} / \mathrm{dia}$ a $11.200 \mathrm{mg} / \mathrm{m}^{2} /$ dia por via oral ${ }^{1}$. Adose máxima diária tem sido de $8.400 \mathrm{mg} / \mathrm{m}^{2} /$ dia.
Com base nos resultados obtidos em nossos experimentos e na literatura médica, está sendo desenvolvido o Estudo Fase II do Álcool Perílico em pacientes com gliomas malignos recidivos ${ }^{16}$. O projeto visa tratar 20 pacientes no período de 24 meses, quando procuraremos determinar a toxicidade, a dose máxima tolerada e os efeitos antitumorais dessa substância.

Neste artigo, apresentamos a redução de oligodendroglioma anaplásico recidivado tratado com álcool perílico por via intranasal quatro vezes ao dia.

\section{Conclusão}

$\mathrm{Na}$ última década, avanços em diagnóstico por imagem, técnicas cirúrgicas, diagnóstico histológico, tratamento combinado de radioterapia e quimioterapia têm melhorado o prognóstico dos pacientes com oligodendroglioma e oligodendroglioma anaplásico. A cirurgia contínua sendo o principal tratamento para os pacientes portadores de oligodendrogliomas. Contudo, esses tumores apresentam recidivas apesar do esquema terapêutico multimodal pós-operatório. A caracterização da patogênese molecular dos oligodendrogliomas deverá tornar-se uma prática corrente em futuro próximo, proporcionando uma terapia inteligente com agentes mais efetivos e menos tóxicos. Nosso grupo vem desenvolvendo Estudo Clínico Fase II com o Álcool Perílico, inibidor da isoprenilação e farnesilação da Ras, oncoproteína superexpressa nos gliomas malignos recidivos. Neste artigo, discutimos a ação desse agente na regressão tumoral de oligodendroglioma anaplásico recidivado.

\section{Agradecimentos}

A Paulo Costa Carvalho, pela assistência na elaboração das figuras, à Fundação Euclides da Cunha, da Universidade Federal Fluminense (FEC/UFF) e à Assistência Médica Internacional (AMIL).

\section{Referências}

1. BELANGER JT: Perillyl alcohol: applications in oncology. Altern Med Rev 3:448-57, 1998.

2. BELLO MJ, DE CAMPOS JM, VAQUERO J, RUIZ-BARNES $P$, KUSAK ME, SARASA JL et al.: hRAD54 gene and $1 p$ high-resolution deletion-mapping analyses in oligodendrogliomas. Cancer Genet Cytogenet 116:142-7, 2000. 
3. BELLO MJ, LEONE PE, VAQUERO J, DE CAMPOS JM, KUSAK ME, SARASA JL et al.: Allelic loss at $1 p$ and $19 q$ frequently occurs in association and may represent early oncogenic events in oligodendroglial tumors. Int J Cancer 64:207-10, 1995.

4. BONNI A, BRUNET A, WEST AE, DATTA SR, TAKASU MA, GREENBERG ME: Cell survival promoted by the RasMAPK signaling pathway by transcription-dependent and -independent mechanisms. Science 12:1358-62, 1999.

5. BROHOLM H, BOLS B, HEEGAARD S, BRAENDSTRUP O: Immunohistochemical investigation of $\mathrm{p} 53$ and EGFR expression of oligodendrogliomas. Clin Neuropathol 18:17680, 1999.

6. BURKE YD, AYOUBI AS, WERNER SR, MCFARLAND BC, HEILMAN DK, RUGGERI BA et al.: Effects of the isoprenoids perillyl alcohol and farnesol on apoptosis biomarkers in pancreatic cancer chemoprevention. Anticancer Res 22:3127-34, 2002

7. CAIRNCROSS JG, MACDONALD DR: Successful chemotherapy for recurrent malignant oligodendroglioma. Ann Neurol 23:360-4, 1988.

8. CHINOT O: Chemotherapy for the treatment of oligodendroglial tumors. Semin Oncol 28:13-8, 2001.

9. CHUNG BH, LEE HY, LEE JS, YOUNG CY: Perillyl alcohol inhibits the expression and function of the androgen receptor in human prostate cancer cells. Cancer Lett Jul 16, 2005.

10. COONS SW, JOHNSON PC, SCHEITHAUER BW, YATES AJ, PEARL DK: Improving diagnostic accuracy and interobserver concordance in the classification and grading of primary gliomas. Cancer 79:1381-93, 1997.

11. DEHGHANI F, SCHACHENMAYR W, LAUN A, KORF HW: Prognostic implication of histopathological, immunohistochemical and clinical features of oligodendrogliomas: a study of 89 cases. Acta Neuropathol 95:493-504, 1998.

12. ENGELHARD HH, STELEA A, COCHRAN EJ: Oligodendroglioma: pathology and molecular biology. Surg Neurol 58:111-7; 2002.

13. ENGELHARD HH, STELEA A, MUNDT A: Oligodendroglioma and anaplastic oligodendroglioma: clinical features, treatment, and prognosis. Surg Neurol 60:443-56, 2003.

14. FISCHER JSG, SILVA MMS, CARVALHO PC, PASCHOAL MP, GATTASS CR, CARVALHO MGC: Efeito do álcool perílico na expressão gênica de células de adenocarcinoma de pulmão humano. J Bras Pneumol 31:373-81, 2005.

15. FONSECACO: Patogênese molecular e estratégia terapêutica dos gliomas. Rio de Janeiro: DiLivros/EdUFF, 2005.

16. FONSECA CO, LANDEIRO JA, CLARK SS, QUIRICOSANTOS T, DA COSTA CARVALHO MDA G, GATTASS CR: Recent advances in the molecular genetics of malignant gliomas disclose targets for antitumor agent perillyl alcohol. Surg Neurol 65 (Suppl 1):S2-8, 2006.

17. FORTIN D, CAIRNCROSS GJ, HAMMOND RR: Oligodendroglioma: an appraisal of recent data pertaining to diagnosis and treatment. Neurosurgery 45:1279-91, 1999.

18. FORTIN D, MACDONALD DR, STITT L, CAIRNCROSS JG: PCV for oligodendroglial tumors: in search of prognostic factors for response and survival. Can J Neurol Sci 28:215-23, 2001.

19. GANNETT DE, WISBECK WM, SILBERGELD DL, BERGER MS: The role of postoperative irradiation in the treatment of oligodendroglioma. Int J Radiat Oncol Biol Phys 30:567-73, 1994.

20. HAAG JD, GOULD MN: Mammary carcinoma regression induced by perillyl alcohol, a hydroxylated analog of limonene. Cancer Chemother Pharmacol 34:477-83, 1994.
21. HENDERSON KH, SHAW EG: Randomized trials of radiation therapy in adult low-grade gliomas. Semin Radiat Oncol 11:145-51, 2001.

22. HOANG-XUAN K, CAPELLE L, KUJAS M, TAILLIBERT $S$, DUFFAU H, LEJEUNE $J$ et al.: Temozolomide as initial treatment for adults with low-grade oligodendrogliomas or oligoastrocytomas and correlation with chromosome $1 \mathrm{p}$ deletions. J Clin Oncol 22:3133-8, 2004.

23. HOANG-XUAN K, HE J, HUGUET S, MOKHTARI K, MARIE Y, KUJAS M et al.: Molecular heterogeneity of oligodendrogliomas suggests alternative pathways in tumor progression. Neurology 57:1278-81, 2001.

24. HUANG Cl: Oligodendroglioma occurring after radiation therapy for pituitary adenoma. J Neurol Neurosurg Psychiatry 50:1619-24, 1987

25. HUSEMANN K, WOLTER M, BUSCHGES R, BOSTROM J SABEL M, REIFENBERGER G: Identification of two distinct deleted regions on the short arm of chromosome 1 and rare mutation of the CDKN2C gene from 1p32 in oligodendroglial tumors. J Neuropathol Exp Neurol 58:1041-50, 1999.

26. JEREMIC B, SHIBAMOTO Y, GRUIJICIC D, MILICIC B, STOJANOVIC M, NIKOLIC $N$ et al. Combined treatment modality for anaplastic oligodendroglioma: a phase II study. J Neurooncol 43:179-85, 1999.

27. KLEIHUES P, CAVENEE WK: Pathology and genetics of tumors of the nervous system. ED 2. Lyon, IARC Press, 2000.

28. KLEIHUES P, LOUIS DN, SCHEITHAUER BW, RORKE LB, REIFENBERGER G, BURGER PC et al.: The WHO classification of tumors of the nervous system. J Neuropathol Exp Neurol 61: 215-25, 2002.

29. KROS JM, LIE ST, STEFANKO SZ: Familial occurrence of polymorphous oligodendrogliomas. Neurosurgery 34:732-6; 1994

30. KYRITSIS AP, YUNG WK, BRUNER J, GLEASON MJ, LEVIN VA: The treatment of anaplastic oligodendrogliomas and mixed gliomas. Neurosurgery 32:365-70, 1993.

31. LEMAIRE JJ, KHALIL T, BARD JJ, VERRELLE P: Oligodendrogliomas and radiosurgery. Neurochirurgie 51:393-9, 2005.

32. LEONARDI MA, LUMENTA CB: Oligodendrogliomas in the CT/MR-era. Acta Neurochir (Wien) 143:1195-203, 2001.

33. LEVIN VA: Controversies in the treatment of low-grade astrocytomas and oligodendrogliomas. Curr Opin Oncol 8:175-7, 1996.

34. LUDWIG CL, SMITH MT, GODFREY AD, ARMBRUSTMACHER VW: A clinicopathological study of 323 patients with oligodendrogliomas. Ann Neurol 19:15-21, 1986.

35. LURIA-PREVATT M, MORREALE J, GREGUS J, ALBERTS DS, KAPER F, GIACCIA A et al.: Effects of perillyl alcohol on melanoma in the TPras mouse model. Cancer Epidemiol Biomarkers Prev 11:573-9, 2002.

36. MCLENDON RE, WIKSTRAND CJ, MATTHEWS MR, ALBARADEI R, BIGNER SH, BIGNER DD: Glioma-associated antigen expression in oligodendroglial neoplasms. Tenascin and epidermal growth factor receptor. J Histochem Cytochem 48:1103-10, 2000.

37. MILLS JJ, CHARI RS, BOYER IJ, GOULD MN, JIRTLE RL: Induction of apoptosis in liver tumors by the monoterpene perillyl alcohol. Cancer Res 55:979-83, 1995.

38. MUELLER W, HARTMANN C, HOFFMANN A, LANKSCH W, KIWIT J, TONN J et al.: Genetic signature of oligoastrocytomas correlates with tumor location and denotes distinct molecular subsets. Am J Pathol 161:313-9, 2002. 
39. OLSON JD, RIEDEL E, DEANGELIS LM: Long-term outcome of low-grade oligodendroglioma and mixed glioma. Neurology 54:1402-3, 2000.

40. PALEOLOGOS NA, MACDONALD DR, VICK NA, CAIRNCROSS JG: Neoadjuvant procarbazine, CCNU, and vincristine for anaplastic and aggressive oligodendroglioma. Neurology 53:1141-3, 1999.

41. PEREZ-DIAZ C, CABELLO A, LOBATO RD, RIVAS JJ, CABRERA A: Oligodendrogliomas arising in the scar of a brain contusion. Report of two surgically verified cases. Surg Neurol 24:581-6, 1985.

42. PERRY JR, BROWN MT, GOCKERMAN JP: Acute leukemia following treatment of malignant glioma. J Neurooncol 40:39-46, 1998

43. REDDY BS, WANG CX, SAMAHA H, LUBET R, STEELE VE, KELLOFF GJ et al.: Chemoprevention of colon carcinogenesis by dietary perillyl alcohol. Cancer Res 57: 420-5, 1997.

44. ROBINSON S, COHEN M, PRAYSON R, RANSOHOFF RM, TABRIZI N, MILLER RH: Constitutive expression of growthrelated oncogene and its receptor in oligodendrogliomas. Neurosurgery 48: 864-73, 2001.

45. ROWINSKY EK, WINDLE JJ, VON HOFF DD: Ras protein farnesyltransferase: a strategic target for anticancer therapeutic development. J Clin Oncol 17:3631-52, 1999.

46. SHI W, GOULD MN: Induction of differentiation in neuro-2A cells by the monoterpene perillyl alcohol. Cancer Lett 95:1-6, 1995.

47. STARK MJ, BURKE YD, MCKINZIE JH, AYOUBI AS, CROWELL PL: Chemotherapy of pancreatic cancer with the monoterpene perillyl alcohol. Cancer Lett 96:15-21, 1995.

48. STREFFER J, SCHABET M, BAMBERG M, GROTE EH, MEYERMANN R, VOIGT $K$ et al.: A role for preirradiation PCV chemotherapy for oligodendroglial brain tumors. J Neurol 247:297-302, 2000.
49. VAN DEN BENT MJ Chemotherapy of oligodendroglial tumours: current developments. Forum (Genova) 10:108-18, 2000.

50. VAN DEN BENT MJ, KROS JM, HEIMANS JJ, PRONK LC, VAN GROENINGEN CJ, KROUWER HG et al.: Response rate and prognostic factors of recurrent oligodendroglioma treated with procarbazine, $\mathrm{CCNU}$, and vincristine chemotherapy. Neurology 51:1140-5, 1998.

51. VAN DEN BENT MJ, HEGI ME, STUPP R: Recent developments in the use of chemotherapy in brain tumours. Eur $\mathrm{J}$ Cancer [Epub ahead of print].

52. VENINGA T, LANGENDIJK HA, SLOTMAN BJ, RUTTEN EH, VAN DER KOGEL AJ, PRICK MJ et al.: Reirradiation of primary brain tumours: survival, clinical response and prognostic factors. Radiother Oncol 59:127-37, 2001.

53. WESTERGAARD L, GJERRIS F, KLINKEN L: Prognostic factors in oligodendrogliomas. Acta Neurochir (Wien) 139:600-5, 1997.

54. ZLATESCU MC, TEHRANIYAZDI A, SASAKI H, MEGYESI JF, BETENSKY RA, LOUIS DN et al.: Tumor location and growth pattern correlate with genetic signature in oligodendroglial neoplasms. Cancer Res 61:6713-5, 2001.

Original recebido em fevereiro de 2006

Aceito para publicação em setembro de 2006

\section{Endereço para correspondência}

Clovis Orlando da Fonseca

Rua Alberto Bianchi 57 / 101

22795-390 - Rio de Janeiro, RJ

E-mail: clovis.orlando@uol.com.br 